Neurosurg Focus 17 (2):E12, 2004 Click here to return to Table of Contents

\title{
Complete resection of a complex glomus jugulare tumor with extensive venous involvement
}

\author{
Case report
}

\author{
Sujit S. Prabhu, M.D., F.R.C.S.(Ed.), and Franco DeMonte, M.D., F.R.C.S.(C) \\ Department of Neurosurgery, The Brain Tumor Center, The University of Texas M. D. Anderson \\ Cancer Center, Houston, Texas
}

\begin{abstract}
The authors describe a case of a complex glomus jugulare tumor with extensive involvement of the venous system. The entire left internal jugular vein up to its innominate insertion was involved with tumor, with proximal extension to the sigmoid sinus, transverse sinus, and the torcular herophili. Gross-total resection of the tumor required a twostage median sternotomy. This is the first case report of a glomus jugulare tumor in which there was such extensive involvement of the venous system, requiring a multidisciplinary team approach for complete resection.
\end{abstract}

\section{KEY WORDS • glomus jugulare tumor • paraganglioma • skull base tumor}

\section{CASE HISTORY}

This 40-year-old right-handed woman presented with a history of left-sided tinnitus, otalgia, and hearing loss of 5 to 6 years' duration before being examined at The University of Texas M. D. Anderson Cancer Center. Approximately 6 to 7 months before her admission, a left neck mass developed near the IJV. On clinical examination she had no evidence of lower cranial nerve deficits. She had left-sided conductive hearing loss, but the remainder of her examination yielded normal findings.

Admission MR images obtained in October 1994 demonstrated the presence of a sausage-shaped lesion extending from the jugular bulb and involving the sigmoid sinus and the transverse sinus as far as the torcular herophili on the left side (Fig. 1). Inferiorly, the tumor extended into the neck and the innominate vein (Figs. 2A and 3). The lesion was also found to extend into the temporal bone, the mesotympanum, and the middle ear. Cerebral angiography revealed extensive tumor involvement of the left IJV extending below the level of the clavicle (Fig. 2B).

\section{Tumor Management}

The patient underwent a left subtotal temporal bone resection with a posterior fossa craniotomy. The head and neck surgeons performed a modified radical neck dissection and a superficial parotidectomy with a left infratemporal fossa approach to the glomus jugulare tumor. The facial nerve was left in its bone canal. The outer wall of the transverse and sigmoid sinuses as well as the jugular

Abbreviations used in this paper: $\mathrm{CA}=$ carotid artery; IJV = internal jugular vein; $\mathrm{MR}=$ magnetic resonance. bulb were removed to approach the tumor. The anterior wall of the jugular bulb containing the openings of the inferior petrosal sinus was intact. The posterior wall of the transverse sinus was sutured to the anterior wall to close off the structure once the tumor was removed from the torcular herophili. The tumor was then dissected from the posterior adventitial wall of the CA and removed completely from the temporal bone. The neck dissection included resection of the IJV, which was filled with tumor to the level of the clavicle.

Three weeks after the first operation, the patient underwent a median sternotomy to resect the remainder of the tumor, which was present in the IJV and innominate vein. A venotomy in the proximal IJV was made once proximal and distal control of the innominate vein was established. The intravascular tumor was delivered from the innominate vein and the IJV was ligated. Following the initial operation, the patient had experienced a mild seventh cranial nerve palsy, from which she recovered completely. Her last MR imaging study, which was obtained in 2002, demonstrated no evidence of any residual or recurrent lesion. The patient received no adjuvant treatments and the histopathological findings were consistent with a paraganglioma.

\section{DISCUSSION}

Approximately $40 \%$ of glomus jugulare paragangliomas extend into the cranial cavity. ${ }^{2,3}$ These tumors can cause not only progressive hearing loss and a sensation of otic fullness or pounding, but also multiple lower cranial nerve palsies (glomus jugulare syndrome). In "functional" tumors, signs of catecholamine secretion include hyper- 


\section{S. S. Prabhu and F. DeMonte}
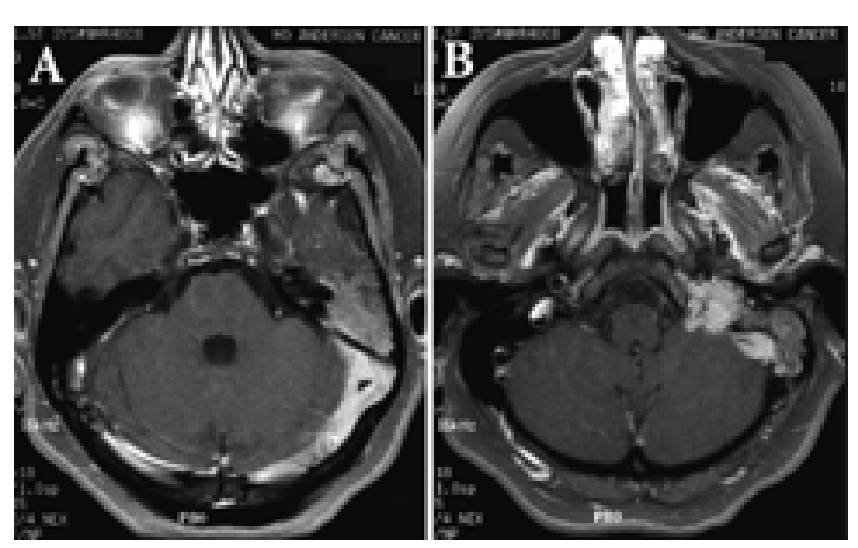

Fig. 1. A and B: Axial $\mathrm{T}_{1}$-weighted $\mathrm{MR}$ images obtained with Gd contrast, demonstrating a left glomus jugulare tumor with involvement of the left sigmoid and transverse sinuses.

tension, palpitation, and sweating. ${ }^{3}$ With rare exceptions, tumors of the glomus jugulare are benign, slow-growing paragangliomas. Although in the past these tumors were deemed unresectable, advances in neuroimaging, skull base techniques, embolization, anesthesia, and emphasis on postoperative care have markedly improved the surgeon's ability to remove these tumors and have improved outcome in an overwhelming number of patients. ${ }^{1}$ Also, studies have shown that total removal has been effective in achieving a cure in a number of cases. ${ }^{4-6}$ Paragangliomas rarely metastasize and the role of radiation therapy in the treatment of residual jugular tympanic paragangliomas is still controversial. Many reports have indicated control rates of 70 to $90 \%$ with radiation as primary, adjunctive, or salvage therapy.

In a recent article on the histological features of jugular foramen and glomus tumors, Sen, et al., ${ }^{7}$ described a dense connective-tissue matrix in the pars nervosa among the cranial nerve fascicles in the jugular foramen. Immedi-

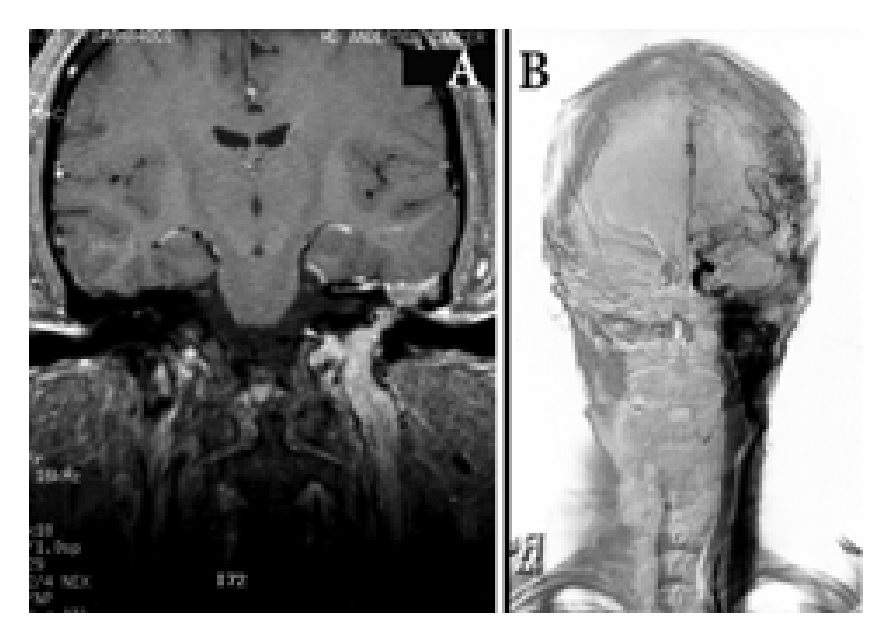

Fig. 2. A: Coronal $\mathrm{T}_{1}$-weighted $\mathrm{MR}$ image of the skull base and upper neck, revealing a sausage-shaped lesion extending from the jugular foramen into the upper neck. B: Cerebral angiogram showing extensive involvement of the left IJV extending below the clavicle on the left side.

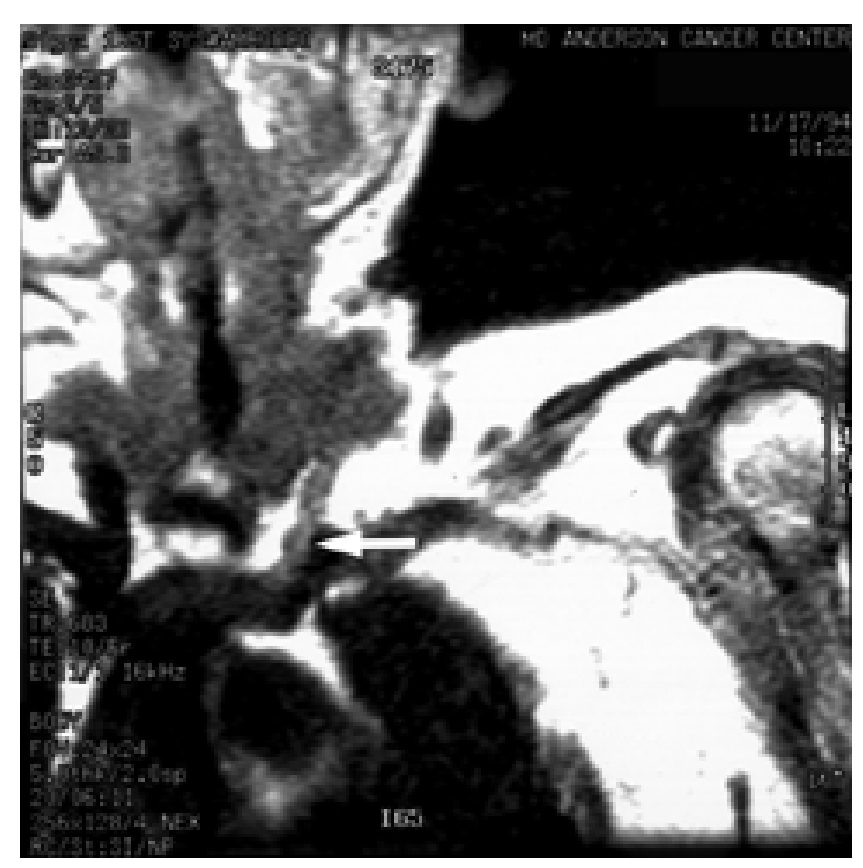

Fig. 3. Coronal MR image of the thoracic inlet. The arrow indicates tumor in the IJV with extension into the innominate vein.

ately outside the foramen, the internal CA, the IJV, and the cranial nerves are surrounded by a common connective tissue sheath. Lesions such as meningiomas and glomus tumors of the temporal bone are not generally encapsulated and tend to infiltrate along these connective tissue planes as well as locally infiltrating the bone.

Glomus tumors are initially intravascular with no disruption of the anterior wall of the jugular bulb. Characteristically, the tumor is fused to the wall of the jugular bulb, but lies free in the lumen of the sigmoid sinus and IJV. These extensions have no attachments to the vessel walls and can generally be removed via a venotomy. These tumors, however, have the tendency to spread to the skull base and its foramina, the CA, and the various venous channels along the paths of least resistance. In tumors in which the anterior wall of the jugular bulb with its openings for the inferior petrosal sinus is intact (as in our patient), resection can be achieved more easily by peeling the tumor off these structures. Once the pars nervosa is infiltrated, these tumors can spread between the fascicles of the lower cranial nerves and complete resection is not possible without significant lower cranial nerve impairment.

We describe a complex glomus jugulare tumor with extensive involvement of the venous system, including the sigmoid sinus, transverse sinus, and IJV, with extension into the left innominate vein (Fig. 1). The extensive involvement of the venous anatomy has not been previously described in the literature. At surgery, however, the tumor was dissected easily from the posterior adventitial wall of the CA and no significant infiltration of the lower cranial nerves was detected. Because the lower cranial nerves were preserved, our patient's postoperative course was unremarkable. This facilitated a gross-total resection of the tumor from the temporal bone. The thoracic surgeons were successful in obtaining a complete resection 


\section{Total resection of glomus tumor with extensive venous involvement}

of the left IJV below the clavicle with no residual tumor. The resection of these veins did not result in any adverse events. The patient has been disease free over a 10-year follow-up period.

\section{CONCLUSIONS}

We describe a complex glomus jugulare tumor with an unusual degree of vascular extension that was successfully resected. The patient has now been disease free for 10 years after the initial operation.

\section{References}

1. Al-Mefty O, Teixeira A: Complex tumors of the glomus jugulare: criteria, treatment, and outcome. J Neurosurg 97: 1356-1366, 2002

2. Burger PC, Scheithauer BW: Tumors of the Central Nervous System. Washington DC: Armed Forces Institute of Pathology, 1994, pp 317-320
3. Enzinger FM, Weiss SW: Soft Tissue Tumors, ed 3. St. Louis: Mosby, 1995, pp 965-990

4. Forest JA III, Jackson CG, McGrew BM: Long-term control of surgically treated glomus tympanicum tumors. Otol Neurotol 22:232-236, 2001

5. Makek M, Franklin DJ, Zhao JC, et al: Neural infiltration of glomus temporale tumors. Am J Otol 11:1-5, 1990

6. Poe DS, Jackson G, Glasscock ME, et al: Long-term results after lateral cranial base surgery. Laryngoscope 101:372-378, 1991

7. Sen C, Hague K, Kacchara R, et al: Jugular foramen: microscopic anatomic features and implications for neural preservation with reference to glomus tumors involving the temporal bone. Neurosurgery 48:838-848, 2001

Manuscript received June 15, 2004.

Accepted in final form July 19, 2004.

Address reprint requests to: Sujit S. Prabhu, M.D., F.R.C.S.(Ed.), Department of Neurosurgery, Unit 442, The Brain Tumor Center, The University of Texas, M. D. Anderson Cancer Center, Houston, Texas 77030-4009. email: sprabhu@mdanderson.org. 